\title{
Existence and multiplicity of periodic solutions for some second order Hamiltonian systems*
}

\author{
Yiwei Ye Chun-Lei Tang ${ }^{\dagger}$
}

\begin{abstract}
In this paper, we study the existence of nontrivial periodic solutions for the second order Hamiltonian systems $\ddot{u}(t)+\nabla F(t, u(t))=0$, where $F(t, x)$ is either nonquadratic or superquadratic as $|u| \rightarrow \infty$. Furthermore, if $F(t, x)$ is even in $x$, we prove the existence of infinitely many periodic solutions for the general Hamiltonian systems $\ddot{u}(t)+A(t) u(t)+\nabla F(t, u(t))=0$, where $A(\cdot)$ is a continuous $T$-periodic symmetric matrix. Our theorems mainly improve the recent result of Tang and Jiang [X.H. Tang, J. Jiang, Existence and multiplicity of periodic solutions for a class of second-order Hamiltonian systems, Comput. Math. Appl. 59 (2010) 3646-3655].
\end{abstract}

\section{Introduction and main results}

Consider the second order Hamiltonian systems

$$
\left\{\begin{array}{l}
\ddot{u}(t)+\nabla F(t, u(t))=0, \quad \text { a.e. } t \in[0, T], \\
u(0)-u(T)=\dot{u}(0)-\dot{u}(T)=0,
\end{array}\right.
$$

where $T>0, F: \mathbb{R} \times \mathbb{R}^{N} \rightarrow \mathbb{R}$ satisfies the following assumption:

\footnotetext{
${ }^{*}$ Supported by the National Natural Science Foundation of China (No. 11471267).

${ }^{\dagger}$ Corresponding author.

Received by the editors in November 2013.

Communicated by J. Mawhin.

2010 Mathematics Subject Classification : 34C25; 34A34; 37J45.

Key words and phrases : Second-order Hamiltonian systems; Periodic solutions; Mountain pass theorem; Fountain Theorem.
} 
(A) $F(t, x)$ is measurable in $t$ for every $x \in \mathbb{R}^{N}$ and continuously differentiable in $x$ for a.e. $t \in[0, T]$, and there exist $a \in C\left(\mathbb{R}^{+}, \mathbb{R}^{+}\right), b \in L^{1}\left(0, T ; \mathbb{R}^{+}\right)$such that

$$
|F(t, x)| \leq a(|x|) b(t), \quad|\nabla F(t, x)| \leq a(|x|) b(t)
$$

for all $x \in \mathbb{R}^{N}$ and a.e. $t \in[0, T]$.

There has been a vast literature on the study of periodic solutions for Hamiltonian systems via critical point theory since Rabinowitz published his pioneer paper [12] in 1978, see [1-27] and the references therein. We refer to some representative works of them. In [12], Rabinowitz studied the existence of periodic solutions of problem (1.1) under the superquadratic condition:

(AR) There exist $\mu>2$ and $L>0$ such that

$$
0<\mu F(t, x) \leq(\nabla F(t, x), x), \quad \forall t \in[0, T] \text { and }|x| \geq L
$$

Observe that (AR) plays an important role to show that every Palais-Smale sequence for the Lagrangian functional associated with problem (1.1) is bounded. Such condition was first introduced by Ambrosetti and Rabinowitz [1], and are useful in solving superlinear problems such as elliptic equations, Dirac equations and wave equations. Fei [4] considered a kind of new superquadratic assumptions which include some potentials like

$$
F(t, x)=|x|^{2}\left(\ln \left(1+|x|^{p}\right)\right)^{q}, \quad p, q>1 .
$$

He assumed that

$\left(F^{*}\right)$ There exist $\beta>1,1<\lambda<1+(\beta-1) / \beta, c_{1}, c_{2}>0$ and $L>0$ such that

$$
\begin{aligned}
& |\nabla F(t, x)| \leq c_{1}|x|^{\lambda}, \quad \forall t \in[0, T] \text { and }|x| \geq L, \\
& (\nabla F(t, x), x)-2 F(t, x) \geq c_{2}|x|^{\beta}, \quad \forall t \in[0, T] \text { and }|x| \geq L .
\end{aligned}
$$

See also [6, 11, 16, 17, 20] for the related results. Tang and $\mathrm{Wu}[15]$ extended a theorem established by A.C. Lazer, E.M. Landesman and D.R. Meyers [8] on the existence of critical points without compactness assumptions, using the reduction method, the perturbation argument and the least action principle. As an application, they studied the existence of periodic solutions of problem (1.1) with subquadratic and convex potentials, which unifies and generalizes the results in $[10,18,25,26]$. Chen and Ma [3] investigated the general second order Hamiltonian systems

$$
\ddot{u}(t)+A(t) u(t)+\nabla F(t, u(t))=0, \quad \forall t \in \mathbb{R},
$$

where $A(\cdot)$ is a continuous T-periodic symmetric matrix. Applying generic superquadratic conditions instead of (AR) and assuming 0 lies in a gap of $\sigma\left(-d^{2} / d t^{2}-A(t)\right)$, they proved the existence of ground state periodic solutions of problem (1.2), i.e., solutions correspond to the least energy of the action functional of (1.2). The proof relied on a generalized weak linking theorem for strongly 
indefinite problem developed by Schechter and Zou [28]. Li, Su and Tian [9] studied the second order Hamiltonian systems of the form

$$
\ddot{u}(t)+A(t) u(t)=-\lambda u(t)-\nabla F(t, u(t)), \quad t \in \mathbb{R},
$$

where $\lambda \in \mathbb{R}$ is a parameter, $F \in C^{2}\left(\mathbb{R} \times \mathbb{R}^{N}, \mathbb{R}\right)$ is periodic in the first variable and satisfies (AR) condition. Combining Morse theory, topological linking and bifurcation arguments, they established the existence of at least two or three nontrivial $2 \pi$-solutions of $(H S)_{\lambda}$. Schechter [13] dealt with the existence of periodic solutions for the nonautonomous systems

$$
\ddot{u}(t)+\beta \nabla F(t, u(t))=0,
$$

taking advantage of the theory of sandwich pairs (see [29]) and Struwe's monotonicity trick (see [30]). Assuming very weak conditions on $F$, e.g., $\nabla F \in C\left(\mathbb{R} \times \mathbb{R}^{N}, \mathbb{R}\right), 0 \leq \int_{0}^{T} F(t, x) d t \rightarrow+\infty$ as $|x| \rightarrow \infty$, the existence result was obtained for almost all $\beta \leq 6 m^{2} /(\alpha T)$, where $\alpha$ and $m$ are constants satisfying $\int_{0}^{T} F(t, x) d t \leq \alpha$ for all $|x| \leq m$. As for results on the existence of infinitely many solutions of problem (1.1), we refer the readers to [2, 5, 14, 21, 22, 27].

Our motivation mainly comes from the papers $[14,24]$. The existence of solutions of problem (1.1) was considered in Zhao et al. [24] in the case that $F(t, x)=$ $-K(t, x)+W(t, x), K$ satisfies the "pinching" condition introduced by Izydorek and Janczewska [31], i.e.,

$\left(K_{1}\right)$ There exist constants $b_{1}, b_{2}>0$ such that $b_{1}|x|^{2} \leq K(t, x) \leq b_{2}|x|^{2}$ for all $(t, x) \in[0, T] \times \mathbb{R}^{N}$;

and $W$ is asymptotically quadratic at infinity. Precisely, they made the following hypotheses:

$\left(K_{2}\right) K(t, x) \leq(\nabla K(t, x), x) \leq 2 K(t, x)$ for all $(t, x) \in[0, T] \times \mathbb{R}^{N}$.

$\left(W_{1}\right) \nabla W(t, x)=o(|x|)$ as $|x| \rightarrow 0$ uniformly for $t \in[0, T]$.

$\left(W_{2}\right)$ There exists a function $V_{\infty} \in L^{\infty}(0, T ; \mathbb{R})$ such that $\lim _{|x| \rightarrow \infty} \mid \nabla W(t, x)-$ $V_{\infty}(t) x|/| x \mid=0$ uniformly for $t \in[0, T]$, and $\inf _{t \in[0, T]} V_{\infty}(t)>$ $\left(4 \pi^{2}+T^{2}\right) \max \left\{1,2 b_{2}\right\} /\left(T^{2}\right)$.

$\left(W_{3}\right) \widetilde{W}(t, x):=\frac{1}{2}(\nabla W(t, x), x)-W(t, x) \rightarrow+\infty$ as $|x| \rightarrow \infty$ uniformly for $t \in[0, T]$.

Applying Rabinowitz's mountain pass theorem, they obtained the following theorem.

Theorem A (see [24, Theorem 1.1]). Suppose that $F=-K+W, K, W \in$ $C^{1}\left([0, T] \times \mathbb{R}^{N}, \mathbb{R}\right)$ satisfy $\left(K_{1}\right)-\left(K_{2}\right)$ and $\left(W_{1}\right)-\left(W_{3}\right)$. Then problem (1.1) has at least one nontrivial T-periodic solution.

Recently, Tang and Zhang [14] improved Theorem A to more general potentials. They assumed that: 
$\left(K_{3}\right)$ There exist constants $b>0$ and $r \in(1,2]$ such that $K(t, 0)=0$, and $K(t, x) \geq$ $b|x|^{r}$ for all $(t, x) \in[0, T] \times \mathbb{R}^{N}$.

$\left(K_{4}\right)(\nabla K(t, x), x) \leq 2 K(t, x)$ for all $(t, x) \in[0, T] \times \mathbb{R}^{N}$.

$\left(W_{4}\right) \lim \sup _{|x| \rightarrow 0} W(t, x) /|x|^{2}<b$ uniformly for $t \in[0, T]$.

$\left(W_{5}\right)$ There exist constants $a>0$ and $d>0$ such that $W(t, x) \leq a|x|^{2}+d$ for all $(t, x) \in[0, T] \times \mathbb{R}^{N}$.

$\left(W_{6}\right)$ There exists $g \in L^{1}(0, T ; \mathbb{R})$ such that $(\nabla W(t, x), x)-2 W(t, x) \geq g(t)$ for all $(t, x) \in[0, T] \times \mathbb{R}^{N}$, and $\lim _{|x| \rightarrow \infty}[(\nabla W(t, x), x)-2 W(t, x)]=+\infty$ for a.e. $t \in[0, T]$.

$\left(W_{7}\right)$ There exists $x_{0} \in \mathbb{R}^{N}$ such that $\int_{0}^{T}\left[K\left(t, x_{0}\right)-W\left(t, x_{0}\right)-\frac{g(t)}{2}\right] d t<0$.

Theorem B (see [14, Theorem 1.1]). Suppose that $F=-K+W, K$, $W \in C^{1}\left([0, T] \times \mathbb{R}^{N}, \mathbb{R}\right)$ satisfy $\left(K_{3}\right)-\left(K_{4}\right)$ and $\left(W_{4}\right)-\left(W_{7}\right)$. Then problem (1.1) has at least one nontrivial T-periodic solution.

They also considered the situation where $\nabla W$ is superlinear at infinity and proved the following result.

Theorem C (see [24, Theorem 1.2]). Suppose that $F=-K+W, K$, $W \in C^{1}\left([0, T] \times \mathbb{R}^{N}, \mathbb{R}\right)$ satisfy $\left(K_{3}\right)-\left(K_{4}\right),\left(W_{4}\right)$ and

$\left(W_{8}\right)$ There exist $a_{1}, a_{2}>0$ and $v \geq 2$ such that $W(t, x) \leq a_{1}|x|^{v}+a_{2}$ for all $(t, x) \in$ $[0, T] \times \mathbb{R}^{N}$.

$\left(W_{9}\right)$ There exist $a_{3}, a_{4}>0, \mu \geq 2$ and $\mu>v-r$ such that $(\nabla W(t, x), x)-$ $2 W(t, x) \geq a_{3}|x|^{\mu}-a_{4}$ for all $(t, x) \in[0, T] \times \mathbb{R}^{N}$.

Then problem (1.1) has at least one nontrivial T-periodic solution.

If $F(t, x)$ is even in $x$, i.e., $F(t,-x)=F(t, x)$ for all $(t, x) \in[0, T] \times \mathbb{R}^{N}$, they obtained infinitely many solutions by using the symmetric mountain pass theorem.

Theorem D (see [24, Theorem 1.3]). Suppose that $F=-K+W, K, W \in C^{1}([0, T] \times$ $\left.\mathbb{R}^{N}, \mathbb{R}\right)$ satisfy $\left(K_{3}\right)-\left(K_{4}\right),\left(W_{4}\right)$ and $\left(W_{8}\right)-\left(W_{9}\right)$, and that $F$ is even in $x$. Then problem (1.1) has infinitely many T-periodic solutions.

In the present paper, we are interested in nonquadratic and superquadratic potentials. Instead of investigating the properties of $K$ and $W$, we directly impose assumptions on $F$. One nontrivial solution is constructed using the classical mountain pass theorem. Furthermore, if $F$ is even in $x$, we study the existence of infinitely many solutions for the general Hamiltonian systems

$$
\left\{\begin{array}{l}
\ddot{u}+A(t) u(t)+\nabla F(t, u(t))=0, \\
u(0)-u(T)=\dot{u}(0)-\dot{u}(T)=0
\end{array} \quad \text { a.e. } t \in[0, T]\right.
$$


where $A(t)$ is an $N \times N$ symmetric matrix, continuous and T-periodic in $t$. The main results generalize and improve Theorems A-D.

We make the following assumptions:

(F) $\limsup _{|x| \rightarrow 0} \frac{F(t, x)}{|x|^{2}}<0$ uniformly for a.e. $t \in[0, T]$.

$\left(F_{2}\right) \limsup _{|x| \rightarrow \infty} \frac{F(t, x)}{|x|^{2}}<+\infty$ uniformly for a.e. $t \in[0, T]$.

$\left(F_{3}\right)$ There exists $g \in L^{1}(0, T ; \mathbb{R})$ such that

$$
(\nabla F(t, x), x)-2 F(t, x) \geq g(t), \quad \forall(t, x) \in[0, T] \times \mathbb{R}^{N},
$$

and

$$
\lim _{|x| \rightarrow \infty}[(\nabla F(t, x), x)-2 F(t, x)]=+\infty \quad \text { for a.e. } t \in[0, T] .
$$

$\left(F_{3}^{\prime}\right)$ There exists $g \in L^{1}(0, T ; \mathbb{R})$ such that

$$
(\nabla F(t, x), x)-2 F(t, x) \leq g(t), \quad \forall(t, x) \in[0, T] \times \mathbb{R}^{N},
$$

and

$$
\lim _{|x| \rightarrow \infty}[(\nabla F(t, x), x)-2 F(t, x)]=-\infty \quad \text { for a.e. } t \in[0, T] .
$$

$\left(F_{4}\right)$ There exists $x_{0} \in \mathbb{R}^{N}$ such that $\int_{0}^{T} F\left(t, x_{0}\right) d t>0$.

Since $F(t, x)$ may be replaced by $F(t, x)-F(t, 0)$, we may also assume without loss of generality that $F(t, 0)=0$ for all $t \in[0, T]$.

We mention that the nonquadratic type conditions like $\left(F_{3}\right)\left(\right.$ or $\left.\left(F_{3}^{\prime}\right)\right)$ was introduced in Costa and Magalhães [32] for studying the Dirichlet problem $-\Delta u=$ $f(x, u)$ in $\Omega, u=0$ on $\partial \Omega$, where $\Omega \subset \mathbb{R}^{N}$ is smoothly bounded. For Hamiltonian systems, it was used in Jiang and Tang [7] to obtain the existence of periodic and subharmonic solutions for problem (1.1), and in Ma and Zhang [11] for $p$ Laplacian systems.

Theorem 1.1. Assume that $F$ satisfies assumptions $(A)$ and $\left(F_{1}\right)-\left(F_{4}\right)$. Then problem (1.1) has at least one nontrivial solution.

Theorem 1.2. The conclusion of Theorem 1.1 holds if we replace $\left(F_{3}\right)$ by $\left(F_{3}^{\prime}\right)$.

Remark 1.1. Theorem 1.1 generalizes Theorem $B$, and hence also generalizes Theorem A. First, Theorem 1.1 requires the function $F$ satisfies the standard regular assumption $(A)$ instead of being $C^{1}$. Second, it follows from $\left(K_{3}\right)$ and $\left(W_{4}\right)$ that

$$
\limsup _{|x| \rightarrow 0} \frac{F(t, x)}{|x|^{2}}<\limsup _{|x| \rightarrow 0} \frac{-b}{|x|^{2-r}}+b= \begin{cases}0, & \text { if } r=2 \\ -\infty, & \text { if } 1<r<2\end{cases}
$$


which implies $\left(F_{1}\right)$. Moreover, combining $\left(K_{3}\right)$ and $\left(W_{5}\right)$, we have

$$
\limsup _{|x| \rightarrow \infty} \frac{F(t, x)}{|x|^{2}} \leq \limsup _{|x| \rightarrow \infty} \frac{W(t, x)}{|x|^{2}} \leq a<+\infty \quad \text { uniformly for } t \in[0, T],
$$

which gives $\left(F_{2}\right)$. Thirdly, by $\left(K_{4}\right)$ and $\left(W_{6}\right)$, we obtain

$$
\begin{aligned}
(\nabla F(t, x), x)-2 F(t, x) & =[-(\nabla K(t, x), x)+2 K(t, x)]+ \\
& {[(\nabla W(t, x), x)-2 W(t, x)] } \\
& \geq(\nabla W(t, x), x)-2 W(t, x) \\
& \geq g(t)
\end{aligned}
$$

for all $(t, x) \in[0, T] \times \mathbb{R}^{N}$, and

$$
(\nabla F(t, x), x)-2 F(t, x) \geq(\nabla W(t, x), x)-2 W(t, x) \rightarrow+\infty \quad \text { as }|x| \rightarrow \infty
$$

for a.e. $t \in[0, T]$, which implies $\left(F_{3}\right)$. Finally, by $\left(W_{6}\right)$,

$$
-F(t, 0)=K(t, 0)-W(t, 0)=-W(t, 0) \geq \frac{g(t)}{2}, \quad \forall t \in[0, T]
$$

which, together with $\left(W_{7}\right)$, shows that

$$
\begin{aligned}
\int_{0}^{T} F\left(t, x_{0}\right) d t & =\int_{0}^{T}\left[F\left(t, x_{0}\right)-F(t, 0)\right] d t \\
& =\int_{0}^{T}\left(-K\left(t, x_{0}\right)+W\left(t, x_{0}\right)-F(t, 0)\right) d t \\
& \geq \int_{0}^{T}\left(-K\left(t, x_{0}\right)+W\left(t, x_{0}\right)+\frac{g(t)}{2}\right) d t \\
& >0 .
\end{aligned}
$$

Remark 1.2. Theorems 1.1 and 1.2 respectively complement Theorems 4 and 5 in Ma and Zhang [11], where the existence of one nontrivial solution was obtained under conditions $(A),\left(F_{2}\right),\left(F_{3}\right)$ (or $\left(F_{3}^{\prime}\right)$ ) and:

$$
\begin{aligned}
& F(t, x) \geq 0 \text { for all }(t, x) \in[0, T] \times \mathbb{R}^{N} . \\
& \limsup _{|x| \rightarrow 0} F(t, x) /|x|^{2}<2 \pi^{2} / T^{2}<\liminf _{|x| \rightarrow \infty} F(t, x) /|x|^{2}
\end{aligned}
$$

uniformly for a.e. $t \in[0, T]$.

There are functions $F$ which match our Theorem 1.1 but not satisfying Theorems A, B and the results in $[3,4,6,7,11,12,16,17,19,20,23]$. For example, let

$$
F(t, x)= \begin{cases}|x|^{2}-1, & |x|<1 \\ 2|x|^{2}-\ln |x|^{2}-2, & |x| \geq 1 .\end{cases}
$$


Theorem 1.3. Assume that $F$ satisfies assumptions $(A),\left(F_{1}\right),\left(F_{4}\right)$ and:

$\left(F_{5}\right)$ There exists $\lambda>2$ such that

$$
\limsup _{|x| \rightarrow \infty} \frac{F(t, x)}{|x|^{\lambda}}<+\infty \quad \text { uniformly for a.e. } t \in[0, T] .
$$

$\left(F_{6}\right)$ There exists $\beta>\lambda-2$ such that

$$
\liminf _{|x| \rightarrow \infty} \frac{(\nabla F(t, x), x)-2 F(t, x)}{|x|^{\beta}}>0 \quad \text { uniformly for a.e. } t \in[0, T] .
$$

Then problem (1.1) possesses at least one nontrivial T-periodic solution.

Remark 1.3. Assumption $\left(F_{4}\right)$ holds for functions $F$ in Theorem C. Indeed, it follows from $\left(W_{9}\right)$ that

$$
\frac{d}{d s}\left(\frac{W(t, s x)}{s^{2}}\right)=\frac{(\nabla W(t, s x), s x)-2 W(t, s x)}{s^{3}} \geq \frac{a_{3} s^{\mu}|x|^{\mu}-a_{4}}{s^{3}}
$$

for all $(t, x) \in[0, T] \times \mathbb{R}^{N}$ and $s>0$. Integrating both sides from 1 to $s(s>1)$ gives

$$
\frac{W(t, s x)}{s^{2}} \geq g(s):= \begin{cases}W(t, x)+\frac{a_{3}}{\mu-2}|x|^{\mu}\left(s^{\mu-2}-1\right)-\frac{a_{4}}{2}\left(1-\frac{1}{s^{2}}\right), & \text { if } \mu>2, \\ W(t, x)+a_{3}|x|^{\mu} \ln s-\frac{a_{4}}{2}\left(1-\frac{1}{s^{2}}\right), & \text { if } \mu=2,\end{cases}
$$

for all $(t, x) \in[0, T] \times \mathbb{R}^{N}$. Hence, for fixed $x_{0} \in \mathbb{R}^{N} \backslash\{0\}$, we obtain

$$
\begin{array}{r}
\frac{1}{s^{2}} \int_{0}^{T} W\left(t, s x_{0}\right) d t \geq \int_{0}^{T} W\left(t, x_{0}\right) d t+\frac{a_{3}}{\mu-2}\left(s^{\mu-2}-1\right)\left|x_{0}\right|^{\mu} T-\frac{a_{4}}{2}\left(1-\frac{1}{s^{2}}\right) T, \\
\text { if } \mu>2,
\end{array}
$$

and

$$
\frac{1}{s^{2}} \int_{0}^{T} W\left(t, s x_{0}\right) d t \geq \int_{0}^{T} W\left(t, x_{0}\right) d t+a_{3}\left|x_{0}\right|^{\mu} T \ln s-\frac{a_{4}}{2}\left(1-\frac{1}{s^{2}}\right) T, \quad \text { if } \mu=2,
$$

which implies that

$$
\frac{1}{s^{2}} \int_{0}^{T} W\left(t, s x_{0}\right) d t \rightarrow+\infty \text { as } s \rightarrow+\infty
$$

when $\mu \geq 2$. Similarly, using $\left(K_{4}\right)$, we have

$$
\frac{1}{s^{2}} \int_{0}^{T} K\left(t, s x_{0}\right) d t \leq \int_{0}^{T} K\left(t, x_{0}\right) d t \leq \max _{t \in[0, T]} K\left(t, x_{0}\right) T \quad \text { for } s>1 .
$$

Combining this with (1.5), we obtain, for $x_{0} \in \mathbb{R}^{N} \backslash\{0\}$ and $s \geq 1$,

$$
\begin{aligned}
\frac{1}{s^{2}} \int_{0}^{T} F\left(t, s x_{0}\right) d t & =\frac{1}{s^{2}} \int_{0}^{T}\left[-K\left(t, s x_{0}\right)+W\left(t, s x_{0}\right)\right] d t \\
& \geq-\max _{t \in[0, T]} K\left(t, x_{0}\right) T+\frac{1}{s^{2}} \int_{0}^{T} W\left(t, s x_{0}\right) d t \\
& \rightarrow+\infty \text { as } s \rightarrow+\infty .
\end{aligned}
$$

Consequently, $\left(W_{9}\right)$, jointly with $\left(K_{4}\right)$, implies $\left(F_{4}\right)$. 
Remark 1.4. Clearly, it follows from (1.5) that the range of $v$ in $\left(W_{8}\right)$ should be $v>2$.

Remark 1.5. Theorem 1.3 extends Theorem C. First, we impose weaker regularity condition on the potential $F$. Second, as stated in Remarks 1.1 and 1.3, $\left(K_{3}\right)$ and $\left(W_{4}\right)$, and $\left(K_{4}\right)$ and $\left(W_{9}\right)$ imply our hypotheses $\left(F_{1}\right)$ and $\left(F_{4}\right)$, respectively. Besides, $\left(F_{5}\right)$ directly follows from $\left(K_{3}\right)$ and $\left(W_{8}\right)$. And finally, using $\left(K_{4}\right)$ and $\left(W_{9}\right)$, we obtain

$$
\begin{aligned}
(\nabla F(t, x), x)-2 F(t, x) & =[-(\nabla K(t, x), x)+2 K(t, x)]+ \\
& {[(\nabla W(t, x), x)-2 W(t, x)] } \\
\geq & (\nabla W(t, x), x)-2 W(t, x) \\
\geq & a_{3}|x|^{\mu}-a_{4}
\end{aligned}
$$

for all $(t, x) \in[0, T] \times \mathbb{R}^{N}$, which implies that $\left(F_{6}\right)$ holds with $\beta \in(\lambda-2, \mu]$. There are functions $F$ which match our Theorem 1.3 but not satisfying Theorems A-C, 1.1, 1.2 and the results in $[3,4,6,7,11,12,15,16,17,19,20,23]$. For example, let

$$
F(t, x)=|x|^{2}\left(\ln \left(\frac{1}{3}|x|^{4}-|x|^{2}+\frac{4}{5}\right)\right), \quad \forall(t, x) \in[0, T] \times \mathbb{R}^{N}
$$

Theorem 1.4. Assume that $F$ satisfies assumptions $(A),\left(F_{1}\right)$ and

$\left(F_{7}\right)$ There exist $m_{1}, m_{2} \geq 0$ such that $F(t, x) \geq-m_{1}|x|^{2}-m_{2}$ for all $(t, x) \in[0, T] \times$ $\mathbb{R}^{N}$.

(F) $F(t, x) /|x|^{2} \rightarrow+\infty$ as $|x| \rightarrow \infty$ for a.e. $t \in[0, T]$.

$\left(F_{9}\right)$ There exist $\sigma \in(0,1)$ and $L>0$ such that

$$
(\nabla F(t, x), x) \leq a \mathcal{F}(t, x)|x|^{2-\sigma}, \quad \forall t \in[0, T] \text { and }|x| \geq L,
$$

where $\mathcal{F}(t, x)=\frac{1}{2}(\nabla F(t, x), x)-F(t, x)$.

Then problem (1.1) has at least one nontrivial T-periodic solution.

Remark 1.6. If $F \in C^{1}\left(\mathbb{R} \times \mathbb{R}^{N}, \mathbb{R}\right)$ satisfies (AR), then the conditions $\left(F_{7}\right)-\left(F_{9}\right)$ hold. There are functions $F$ which match $\left(F_{7}\right)-\left(F_{9}\right)$, but not satisfying (AR). For example, let

$$
F(t, x)=g(t)|x|^{2} \ln \left(1+|x|^{2}\right), \quad \forall(t, x) \in[0, T] \times \mathbb{R}^{N},
$$

where $g \in L^{1}\left(0, T ; \mathbb{R}^{+}\right)$with $g(t)>0$ for a.e. $t \in[0, T]$.

When $F(t, x)$ is symmetric in $x$, we obtain the existence of infinitely many nontrivial periodic solutions for the general Hamiltonian systems (1.3).

Theorem 1.5. Assume that assumptions $(A),\left(F_{5}\right)-\left(F_{8}\right)$ are satisfied and that $F(t, x)$ is even in $x$. Then problem (1.3) possesses infinitely many solutions. 
Remark 1.7. Theorem 1.5 sharply improves Theorem D, which is the special case of our result corresponding to $A=0$. Furthermore, compared with Theorem $\mathrm{D}$, our assumptions are much weaker. According to Remark 1.5, $\left(K_{3}\right)-\left(K_{4}\right)$ and $\left(W_{8}\right)-\left(W_{9}\right)$ imply the ones $\left(F_{5}\right)$ and $\left(F_{6}\right)$. We shall show that $\left(F_{7}\right)-\left(F_{8}\right)$ still hold under $\left(K_{4}\right)$ and $\left(W_{9}\right)$. From $\left(K_{4}\right)$, for fixed $(t, x) \in[0, T] \times \mathbb{R}^{N}$, the mapping $s \longmapsto K(t, s x) / s^{2}$ is nonincreasing on $(0,+\infty)$, which implies that

$$
K(t, x) \leq K(t, x /|x|)|x|^{2} \leq b_{1}|x|^{2}, \quad \forall t \in[0, T] \text { and }|x| \geq 1,
$$

where $b_{1}=\max _{t \in[0, T],|x|=1} K(t, x)$. Hence,

$$
K(t, x) \leq b_{1}|x|^{2}+b_{2}, \quad \forall(t, x) \in[0, T] \times \mathbb{R}^{N},
$$

where $b_{2}=\max _{t \in[0, T],|x| \leq 1} K(t, x)$. On the other hand, integrating (1.4) from $s$ $(s \in(0,1))$ to 1 , we obtain

$W(t, x) \geq g_{1}(s):= \begin{cases}W(t, s x) s^{-2}+\frac{a_{3}}{\mu-2}|x|^{\mu}\left(1-s^{\mu-2}\right)-\frac{a_{4}}{2}\left(\frac{1}{s^{2}}-1\right), & \text { if } \mu>2, \\ W(t, s x) s^{-2}+a_{3}|x|^{\mu} \ln s^{-1}-\frac{a_{4}}{2}\left(\frac{1}{s^{2}}-1\right), & \text { if } \mu=2,\end{cases}$

which yields that

$$
\begin{aligned}
W(t, x) \geq & W(t, x /|x|)|x|^{2}+\min \left\{\frac{a_{3}}{\mu-2}\left(|x|^{\mu}-|x|^{2}\right), a_{3}|x|^{\mu} \ln |x|\right\} \\
& -\frac{a_{4}}{2}\left(|x|^{2}-1\right) \\
\geq & \left(b_{3}-\frac{a_{4}}{2}\right)|x|^{2}
\end{aligned}
$$

for all $t \in[0, T]$ and $|x|>1$, where $b_{3}=\min _{t \in[0, T],|x|=1} W(t, x)$. Thus

$$
W(t, x) \geq\left(b_{3}-\frac{a_{4}}{2}\right)|x|^{2}-b_{4}, \quad \forall(t, x) \in[0, T] \times \mathbb{R}^{N},
$$

where $b_{4}=\left(b_{3}-\frac{a_{4}}{2}\right)+\max _{t \in[0, T],|x| \leq 1} W(t, x)$. This, jointly with (1.7), shows that

$$
\begin{aligned}
& F(t, x)=-K(t, x)+W(t, x) \geq\left(b_{3}-\frac{a_{4}}{2}-b_{1}\right)|x|^{2}-\left(b_{2}+b_{4}\right), \\
& \forall(t, x) \in[0, T] \times \mathbb{R}^{N},
\end{aligned}
$$

that is, $\left(F_{7}\right)$ holds with $m_{1}=\left(b_{3}-\frac{a_{4}}{2}-b_{1}\right)$ and $m_{2}=b_{2}+b_{4}$. In addition, combining (1.6) and (1.8), we obtain

$$
\frac{F(t, x)}{|x|^{2}}=-\frac{K(t, x)}{|x|^{2}}+\frac{W(t, x)}{|x|^{2}} \geq-b_{1}+\frac{W(t, x)}{|x|^{2}} \rightarrow+\infty \quad \text { as }|x| \rightarrow \infty
$$

for $t \in[0, T]$. Therefore $\left(F_{8}\right)$ holds. 
Remark 1.8. Theorem 1.5 also improves a recent result of Zhang and Liu [22, Theorem 1.2], where the existence of infinitely many solutions was obtained for problem (1.3) under the hypotheses that $F \in C^{1}\left(\mathbb{R} \times \mathbb{R}^{N}, \mathbb{R}\right)$ being $T$-periodic in $t$ and even in $x$, and that

$\left(S Q_{1}\right)$ There exist $a_{1}>0$ and $\lambda>2$ such that $|\nabla F(t, x)| \leq a_{1}\left(1+|x|^{\lambda-1}\right)$ for all $(t, x) \in[0, T] \times \mathbb{R}^{N}$.

$\left(S Q_{2}\right) F(t, x) \geq 0$ for all $(t, x) \in[0, T] \times \mathbb{R}^{N}$, and $\lim _{|x| \rightarrow \infty} F(t, x) /|x|^{2}=+\infty$ uniformly for $t \in[0, T]$.

$\left(S Q_{3}\right)$ There exist $1 \leq \beta \in(\lambda-2,+\infty)$ and $b>0$ such that

$$
\liminf _{|x| \rightarrow \infty} \frac{(\nabla F(t, x), x)-2 F(t, x)}{|x|^{\beta}} \geq b \quad \text { uniformly for } t \in[0, T] .
$$

Obviously, $\left(S Q_{2}\right)$ is stronger than the ones $\left(F_{7}\right)-\left(F_{8}\right)$, and the constants $\beta$ has more freedom in $\left(F_{6}\right)$ than in $\left(S_{3}\right)$. There are functions $A$ and $F$ which match our Theorem 1.5 , but not satisfying Theorem $D$ and the results in $[2,5,22,27]$. For example, let $A(t)$ be the unit matrix of order $N$, and

$$
F(t, x)= \begin{cases}|x|^{2}-1, & |x|<1 \\ |x|^{2} \ln |x|^{2}, & |x| \geq 1\end{cases}
$$

Theorem 1.6. Assume that assumptions $(A),\left(F_{7}\right)-\left(F_{9}\right)$ are satisfied and that $F(t, x)$ is even in $x$. Then problem (1.3) possesses infinitely many solutions.

Remark 1.9. There are functions $F$ which matches our Theorem 1.6 but not satisfying Theorems D, 1.5, and the results in $[2,5,22,27]$. For example, let

$$
F(t, x)=\left|\sin \frac{2 \pi}{T} t\right||x|^{2} \ln \left(1+|x|^{2}\right), \quad \forall(t, x) \in[0, T] \times \mathbb{R}^{N}
$$

The paper is organized as follows. In Section 2, we deal with the existence of periodic solutions of problem (1.1). Theorems 1.1-1.4 are proved by using the mountain pass theorem. Section 3 is concerned with the existence of infinitely many solutions of problem (1.3) and the proofs of Theorems 1.5 and 1.6 are given.

Notation. Throughout the paper we denote by $c$ the various positive constants which may vary from line to line and are not essential to the problem.

\section{Proofs of Theorems 1.1-1.4}

Let $H_{T}^{1}$ be a Hilbert space defined by

$$
H_{T}^{1}=\left\{\begin{array}{l|l}
u:[0, T] \rightarrow \mathbb{R}^{N} & \begin{array}{l}
u \text { is absolutely continuous, } \\
u(0)=u(T) \text { and } \dot{u} \in L^{2}\left(0, T ; \mathbb{R}^{N}\right)
\end{array}
\end{array}\right\}
$$


with the norm given by

$$
\|u\|=\left(\int_{0}^{T}|u(t)|^{2} d t+\int_{0}^{T}|\dot{u}(t)|^{2} d t\right)^{1 / 2} .
$$

In view of [10, Proposition 1.3], for $u \in \widetilde{H}_{T}^{1}=\left\{u \in H_{T}^{1}: \int_{0}^{T} u(t) d t=0\right\}$, we have

$$
\|u\|_{\infty}^{2} \leq \frac{T}{12} \int_{0}^{T}|\dot{u}(t)|^{2} d t \quad \text { (Sobolev's inequality) }
$$

which implies that

$$
\|u\|_{\infty} \leq C\|u\|
$$

for all $u \in H_{T}^{1}$ and some $C>0$, where $\|u\|_{\infty}=\max _{t \in[0, T]}|u(t)|$. It follows that the functional $\varphi$ on $H_{T}^{1}$ given by

$$
\varphi(u)=\frac{1}{2} \int_{0}^{T}|\dot{u}(t)|^{2} d t-\int_{0}^{T} F(t, u(t)) d t
$$

is continuously differentiable and weakly lower semi-continuous on $H_{T}^{1}$, and

$$
\left\langle\varphi^{\prime}(u), v\right\rangle=\int_{0}^{T}(\dot{u}(t), \dot{v}(t)) d t-\int_{0}^{T}(\nabla F(t, u(t)), v(t)) d t
$$

for all $u, v \in H_{T}^{1}$. It is well known that the weak solutions of problem (1.1) correspond to the critical points of $\varphi$ (see [10]).

In order to find the critical points of $\varphi$, we shall show that $\varphi$ satisfies the Cerami condition $(\mathrm{C})$, i.e., $\left(u_{n}\right) \subset E$ has a convergent subsequence whenever $\left\{\varphi\left(u_{n}\right)\right\}$ is bounded and $\left(1+\left\|u_{n}\right\|\right)\left\|\varphi^{\prime}\left(u_{n}\right)\right\| \rightarrow 0$ as $n \rightarrow \infty$.

Lemma 2.1. If conditions $(A),\left(F_{2}\right)$ and $\left(F_{3}\right)$ hold, then $\varphi$ satisfies the $(C)$ condition.

Proof. Suppose that $\left(u_{n}\right) \subset H_{T}^{1}$ is a (C) sequence. Then we have

$$
c \geq 2 \varphi\left(u_{n}\right)-\left\langle\varphi^{\prime}\left(u_{n}\right), u_{n}\right\rangle=\int_{0}^{T}\left[\left(\nabla F\left(t, u_{n}\right), u_{n}\right)-2 F\left(t, u_{n}\right)\right] d t
$$

for all $n \in \mathbb{N}$. As in [7], we claim that $\left(u_{n}\right)$ is bounded. For otherwise, there exists a subsequence, still denoted by $\left(u_{n}\right)$, such that $\left\|u_{n}\right\| \rightarrow \infty$ as $n \rightarrow \infty$. Setting $v_{n}=u_{n} /\left\|u_{n}\right\|$, then $\left\|v_{n}\right\|=1$. Up to a subsequence, we can assume that

$$
\begin{array}{ll}
v_{n} \rightarrow v & \text { weakly in } H_{T}^{1} \\
v_{n} \rightarrow v & \text { strongly in } C\left(0, T ; \mathbb{R}^{N}\right) .
\end{array}
$$

By assumptions $(A)$ and $\left(F_{2}\right)$, there exist constants $d_{1}, L_{1}>0$ such that

$$
F(t, x) \leq d_{1}|x|^{2}+\max _{s \in\left[0, L_{1}\right]} a(s) b(t), \quad \forall x \in \mathbb{R}^{N} \text { and a.e. } t \in[0, T] .
$$


Hence we obtain

$$
\begin{aligned}
\varphi\left(u_{n}\right) & =\frac{1}{2} \int_{0}^{T}\left|\dot{u}_{n}(t)\right|^{2} d t-\int_{0}^{T} F\left(t, u_{n}(t)\right) d t \\
& \geq \frac{1}{2}\left\|u_{n}\right\|^{2}-\left(\frac{1}{2}+d_{1}\right) \int_{0}^{T}\left|u_{n}\right|^{2} d t-\max _{s \in\left[0, L_{1}\right]} a(s) \int_{0}^{T} b(t) d t .
\end{aligned}
$$

Dividing both sides by $\left\|u_{n}\right\|^{2}$ and letting $n \rightarrow \infty$, we get, using (2.4),

$$
0 \geq \frac{1}{2}-\left(\frac{1}{2}+d_{1}\right) \int_{0}^{T}|v(t)|^{2} d t
$$

which implies that $v \neq 0$. Set

$$
E_{1}=\{t \in[0, T]:(\nabla F(t, x), x)-2 F(t, x) \rightarrow+\infty \text { as }|x| \rightarrow \infty\}
$$

and $E_{2}=\{t \in[0, T]: v(t) \neq 0\}$. Then one has $E_{1} \cap E_{2} \neq \varnothing$ and $\left|u_{n}(t)\right| \rightarrow \infty$ for $t \in E_{1} \cap E_{2}$. Therefore, using $\left(F_{3}\right)$ and Fatou's lemma (see [33]),

$$
\begin{aligned}
\lim _{n \rightarrow \infty} \int_{0}^{T} & {\left[\left(\nabla F\left(t, u_{n}\right), u_{n}\right)-2 F\left(t, u_{n}\right)\right] d t } \\
& =\lim _{n \rightarrow \infty} \int_{E_{1} \cap E_{2}}\left[\left(\nabla F\left(t, u_{n}\right), u_{n}\right)-2 F\left(t, u_{n}\right)\right] d t+ \\
& \quad \lim _{n \rightarrow \infty} \int_{[0, T] \backslash\left(E_{1} \cap E_{2}\right)}\left[\left(\nabla F\left(t, u_{n}\right), u_{n}\right)-2 F\left(t, u_{n}\right)\right] d t \\
& \geq \lim _{n \rightarrow \infty} \int_{E_{1} \cap E_{2}}\left[\left(\nabla F\left(t, u_{n}\right), u_{n}\right)-2 F\left(t, u_{n}\right)\right] d t-\int_{0}^{T}|g(t)| d t \\
& =+\infty,
\end{aligned}
$$

a contradiction with (2.3). Hence $\left(u_{n}\right)$ is bounded in $H_{T}^{1}$. Arguing then as in the proof of [10, Proposition 4.1], we conclude that the $(C)$ condition is satisfied. This completes the proof.

Lemma 2.2. If assumptions $(A),\left(F_{5}\right)$ and $\left(F_{6}\right)$ hold, then $\varphi$ satisfies the Cerami condition.

Proof. Let $\left(u_{n}\right)$ be a sequence in $H_{T}^{1}$ such that $\left\{\varphi\left(u_{n}\right)\right\}$ is bounded and $\left(1+\left\|u_{n}\right\|\right)\left\|\varphi^{\prime}\left(u_{n}\right)\right\| \rightarrow 0(n \rightarrow \infty)$. Then we have

$$
\left|\varphi\left(u_{n}\right)\right| \leq c \quad \text { and } \quad\left(1+\left\|u_{n}\right\|\right)\left\|\varphi^{\prime}\left(u_{n}\right)\right\| \leq c
$$

for all $n$. It follows from $\left(F_{6}\right)$ that there exist $d_{2}, L_{2}>0$ such that

$$
(\nabla F(t, x), x)-2 F(t, x) \geq d_{2}|x|^{\beta}, \quad \forall|x| \geq L_{2} \text { and a.e. } t \in[0, T] .
$$

By $(A)$, one has

$$
|(\nabla F(t, x), x)-2 F(t, x)| \leq c_{1} b(t), \quad \forall|x| \leq L_{2} \text { and a.e. } t \in[0, T],
$$

where $c_{1}=\left(L_{2}+2\right) \max _{s \in\left[0, L_{2}\right]} a(s)$. Combining this and (2.6) we get

$$
(\nabla F(t, x), x)-2 F(t, x) \geq d_{2}|x|^{\beta}-d_{2} L_{2}^{\beta}-c_{1} b(t)
$$


for all $x \in \mathbb{R}^{N}$ and a.e. $t \in[0, T]$. Hence we have

$$
\begin{aligned}
c & \geq 2 \varphi\left(u_{n}\right)-\left\langle\varphi^{\prime}\left(u_{n}\right), u_{n}\right\rangle \\
& =\int_{0}^{T}\left[\left(\nabla F\left(t, u_{n}\right), u_{n}\right)-2 F\left(t, u_{n}\right)\right] d t \\
& \geq d_{2} \int_{0}^{T}\left|u_{n}\right|^{\beta} d t-d_{2} L_{2}^{\beta} T-c_{1} \int_{0}^{T} b(t) d t
\end{aligned}
$$

for all $n$, which implies that

$$
\int_{0}^{T}\left|u_{n}\right|^{\beta} d t<+\infty, \quad \forall n \in \mathbb{N}
$$

By assumptions $(A)$ and $\left(F_{5}\right)$, there exist $d_{3}, L_{3}>0$ such that

$$
F(t, x) \leq d_{3}|x|^{\lambda}+a_{L_{3}} b(t)
$$

for all $x \in \mathbb{R}^{N}$ and a.e. $t \in[0, T]$, where $a_{L_{3}}=\max _{s \in\left[0, L_{3}\right]} a(s)$. Combining this with (2.8), (2.5) and Hölder's inequality, we obtain

$$
\begin{aligned}
\frac{1}{2}\left\|u_{n}\right\|^{2} & =\varphi\left(u_{n}\right)+\frac{1}{2} \int_{0}^{T}\left|u_{n}\right|^{2} d t+\int_{0}^{T} F\left(t, u_{n}\right) d t \\
& \leq c+\frac{1}{2} T^{1-\frac{2}{\lambda}}\left(\int_{0}^{T}\left|u_{n}\right|^{\lambda} d t\right)^{\frac{2}{\lambda}}+d_{3} \int_{0}^{T}\left|u_{n}\right|^{\lambda} d t+a_{L_{3}} \int_{0}^{T} b(t) d t
\end{aligned}
$$

for all $n$. If $\beta>\lambda$, Hölder's inequality and (2.7) imply that

$$
\int_{0}^{T}\left|u_{n}\right|^{\lambda} d t \leq T^{\frac{\beta-\lambda}{\beta}}\left(\int_{0}^{T}\left|u_{n}\right|^{\beta} d t\right)^{\frac{\lambda}{\beta}}<+\infty, \quad \forall n \in \mathbb{N}
$$

and hence the boundedness of $\left(u_{n}\right)$ follows from (2.9). If $\beta \leq \lambda$, using (2.1), we have

$$
\int_{0}^{T}\left|u_{n}\right|^{\lambda} d t=\int_{0}^{T}\left|u_{n}\right|^{\beta}\left|u_{n}\right|^{\lambda-\beta} d t \leq\left\|u_{n}\right\|_{\infty}^{\lambda-\beta} \int_{0}^{T}\left|u_{n}\right|^{\beta} d t \leq C^{\lambda-\beta}\left\|u_{n}\right\|^{\lambda-\beta} \int_{0}^{T}\left|u_{n}\right|^{\beta} d t
$$

for all $n$. Noticing that $\lambda-\beta<2,(2.9)$ still deduces that $\left(u_{n}\right)$ is bounded. Arguing then as in the proof of [10, Proposition 4.1], we conclude that the $(C)$ condition is satisfied.

Lemma 2.3. Under assumptions $(A)$ and $\left(F_{7}\right)-\left(F_{9}\right), \varphi$ satisfies the $(C)$ condition.

Proof. Let $\left(u_{n}\right) \subset H_{T}^{1}$ be such that $\left\{\varphi\left(u_{n}\right)\right\}$ is bounded and $\left(1+\left\|u_{n}\right\|\right)\left\|\varphi^{\prime}\left(u_{n}\right)\right\| \rightarrow$ 0 as $n \rightarrow \infty$. We have, for large $n$,

$$
c \geq \varphi\left(u_{n}\right)-\frac{1}{2}\left\langle\varphi^{\prime}\left(u_{n}\right), u_{n}\right\rangle=\int_{0}^{T} \mathcal{F}\left(t, u_{n}\right) d t .
$$


This yields, setting $\Omega_{n}(\alpha, \beta):=\left\{t \in[0, T]|\alpha \leq| u_{n}(t) \mid<\beta\right\}$ for $0 \leq \alpha<\beta$, that

$$
\begin{aligned}
\int_{\Omega_{n}(\beta,+\infty)} \mathcal{F}\left(t, u_{n}\right) d t & \leq c-\int_{\Omega_{n}(0, \beta)} \mathcal{F}\left(t, u_{n}\right) d t \\
& \leq c+\int_{\Omega_{n}(0, \beta)}\left|\frac{1}{2}\left(\nabla F\left(t, u_{n}\right), u_{n}\right)-F\left(t, u_{n}\right)\right| d t \\
& \leq c+(\beta+1) a_{\beta} \int_{0}^{T} b(t) d t
\end{aligned}
$$

for large $n$, where $a_{\beta}=\max _{s \in[0, \beta]} a(s)$. In virtue of [10, Proposition 4.1], it suffices to show that $\left(u_{n}\right)$ is bounded. Arguing indirectly, we may assume that $\left\|u_{n}\right\| \rightarrow \infty$ after passing to a subsequence. Take $v_{n}=u_{n} /\left\|u_{n}\right\|$. Then $\left\|v_{n}\right\|=1$, and going to a subsequence, we can assume that

$$
v_{n} \rightarrow v_{0} \quad \text { weakly in } H_{T}^{1} \text { and } v_{n} \rightarrow v_{0} \text { strongly in } C\left(0, T ; \mathbb{R}^{N}\right) .
$$

Case 1. $v_{0} \neq 0$ in $H_{T}^{1}$. Noting $\left\{\varphi\left(u_{n}\right)\right\}$ is bounded, we get

$$
\int_{0}^{T} \frac{F\left(t, u_{n}\right)}{\left\|u_{n}\right\|^{2}} d t=\frac{1}{2}-\frac{1}{2} \int_{0}^{T}\left|v_{0}\right|^{2} d t+o(1) .
$$

On the other hand, take

$$
E_{3}:=\left\{t \in[0, T]: F(t, x) /|x|^{2} \rightarrow+\infty \text { as }|x| \rightarrow \infty\right\} .
$$

Using $\left(F_{7}\right),\left(F_{8}\right)$ and Fatou's lemma (see [33]), we obtain

$$
\begin{aligned}
\int_{0}^{T} \frac{F\left(t, u_{n}\right)}{\left\|u_{n}\right\|^{2}} d t & =\int_{E_{3} \cap\left\{v_{0} \neq 0\right\}} \frac{F\left(t, u_{n}\right)}{\left|u_{n}\right|^{2}}\left|v_{n}\right|^{2} d t+\int_{[0, T] \backslash\left(E_{3} \cap\left\{v_{0} \neq 0\right\}\right)} \frac{F\left(t, u_{n}\right)}{\left\|u_{n}\right\|^{2}} d t \\
& \geq \int_{E_{3} \cap\left\{v_{0} \neq 0\right\}} \frac{F\left(t, u_{n}\right)}{\left|u_{n}\right|^{2}}\left|v_{n}\right|^{2} d t-m_{1} \int_{0}^{T}\left|v_{n}\right|^{2} d t-\frac{m_{2} T}{\left\|u_{n}\right\|^{2}} \\
& \rightarrow+\infty \text { as } n \rightarrow \infty,
\end{aligned}
$$

a contradiction.

Case 2. $v_{0}=0$ in $H_{T}^{1}$. Noting that

$$
\left\langle\varphi^{\prime}\left(u_{n}\right), u_{n}\right\rangle=\left\|u_{n}\right\|^{2}-\int_{0}^{T}\left|u_{n}\right|^{2} d t-\int_{0}^{T}\left(\nabla F\left(t, u_{n}\right), u_{n}\right) d t
$$

for all $n$, one has, by the second limit of (2.11),

$$
\int_{0}^{T} \frac{\left(\nabla F\left(t, u_{n}\right), u_{n}\right)}{\left\|u_{n}\right\|^{2}} d t \rightarrow 1 \text { as } n \rightarrow \infty .
$$

For any $\varepsilon>0(<1 / 2)$, using $\left(F_{9}\right),(2.10)$ and $(2.1)$, we can choose $\beta_{\varepsilon}>L$ sufficiently large such that

$$
\begin{aligned}
\int_{\Omega_{n}\left(\beta_{\varepsilon},+\infty\right)} \frac{\left(\nabla F\left(t, u_{n}\right), u_{n}\right)}{\left\|u_{n}\right\|^{2}} d t & \leq \int_{\Omega_{n}\left(\beta_{\varepsilon},+\infty\right)} \frac{a \mathcal{F}\left(t, u_{n}\right)\left|u_{n}\right|^{2-\sigma}}{\left\|u_{n}\right\|^{2}} d t \\
& \leq \frac{a\left\|v_{n}\right\|_{\infty}^{2}}{\beta_{\varepsilon}^{\sigma}} \int_{\Omega_{n}\left(\beta_{\varepsilon},+\infty\right)} \mathcal{F}\left(t, u_{n}\right) d t \\
& \leq \frac{c}{\beta_{\varepsilon}^{\sigma}} \\
& <\varepsilon
\end{aligned}
$$


for all $n$. In addition,

$$
\int_{\Omega_{n}\left(0, \beta_{\varepsilon}\right)} \frac{\left(\nabla F\left(t, u_{n}\right), u_{n}\right)}{\left\|u_{n}\right\|^{2}} d t \leq \frac{\beta_{\varepsilon} a_{\beta_{\varepsilon}}}{\left\|u_{n}\right\|^{2}} \int_{0}^{T} b(t) d t<\varepsilon
$$

for $n$ large enough. Combining this with (2.14), we obtain

$$
\int_{0}^{T} \frac{\left(\nabla F\left(t, u_{n}\right), u_{n}\right)}{\left\|u_{n}\right\|^{2}} d t<2 \varepsilon<1 \quad \text { for } n \text { sufficiently large, }
$$

which contradicts (2.13).

For convenience to quote, we state Rabinowitz's mountain pass theorem (see $[34$, Theorem 2.2]). In the linking theorem it is usually assumed that $\Phi$ satisfies the stronger Palais-Smale condition, however, the Cerami condition is sufficient for the deformation lemma (see [35]), and hence for the linking theorem to hold.

Proposition 2.1. Let $E$ be a real Banach space and $\Phi \in C^{1}(E, \mathbb{R})$ satisfies the Cerami condition (C). Suppose that $\Phi(0)=0$ and

(i) There are constants $\rho, \alpha>0$ such that $\left.\Phi\right|_{\partial B_{\rho}(0)} \geq \alpha$, where $B_{\rho}(0)$ is an open ball in $E$ of radius $\rho$ centered at 0 .

(ii) There exists $e \in E \backslash B_{\rho}(0)$ such that $\Phi(e) \leq 0$.

Then $\Phi$ possesses a critical value $c \geq \alpha$ given by

$$
c=\inf _{h \in \Gamma} \max _{s \in[0,1]} \Phi(h(s)),
$$

where $\Gamma=\{h \in C([0,1], E): h(0)=0, h(1)=e\}$.

Proof of Theorem 1.1. In view of Lemma 2.1, the functional $\varphi$ introduced in (2.2) satisfies the $(\mathrm{C})$ condition. It remains to verify $(i)$ and $(i i)$ of Proposition 2.1.

It follows from $\left(F_{4}\right)$ that

$$
\varphi\left(x_{0}\right)=-\int_{0}^{T} F\left(t, x_{0}\right) d t<0=\varphi(0),
$$

which gives (ii) directly. By $\left(F_{1}\right)$, we have, for $\varepsilon \in\left(0,-\lim \sup _{|x| \rightarrow 0} F(t, x) /|x|^{2}\right)$, there exists $\delta>0$ such that

$$
F(t, x) \leq-\varepsilon|x|^{2}, \quad \forall|x| \leq \delta \text { and a.e. } t \in[0, T] .
$$

Thus we obtain, using (2.1),

$$
\varphi(u) \geq \frac{1}{2} \int_{0}^{T}|\dot{u}|^{2} d t+\varepsilon \int_{0}^{T}|u|^{2} d t \geq \min \left\{\frac{1}{2}, \varepsilon\right\}\|u\|^{2}
$$

for all $u \in H_{T}^{1}$ with $\|u\| \leq \min \left\{\delta / C,\left|x_{0}\right| / 2\right\}$. So $(i)$ holds with $\rho=\min \{\delta / C$, $\left.\left|x_{0}\right| / 2\right\}$ and $\alpha=\min \{1 / 2, \varepsilon\} \rho^{2}$. Consequently, by Proposition 2.1, $\varphi$ has one 
nonzero critical point, and hence problem (1.1) has one nontrivial solution. This concludes the proof.

Proof of Theorem 1.2. The proof is similar to that of Theorem 1.1 and is omitted.

Proof of Theorem 1.3. The proof follows the same lines as that of Theorem 1.1 with Lemma 2.1 replaced by Lemma 2.2.

Proof of Theorem 1.4. It follows from Lemma 2.3 that $\varphi$ satisfies the Cerami condition. As in the proof of Theorem $1.1,\left(F_{1}\right)$ implies that, for $\rho:=\delta / C$,

$$
\left.\varphi\right|_{\partial B_{\rho}(0)} \geq \min \left\{\frac{1}{2}, \varepsilon\right\} \frac{\delta^{2}}{C^{2}} .
$$

Choose $u_{0} \in C^{\infty}\left(0, T ; \mathbb{R}^{N}\right)$ such that $\left\|u_{0}\right\|=1,\left|u_{0}(t)\right|=1$ for $t \in[T / 3, T / 2]$ and assume $s>1$. Using $\left(F_{7}\right),\left(F_{8}\right)$ and Fatou's lemma, we obtain

$$
\begin{aligned}
\frac{\varphi\left(s u_{0}\right)}{s^{2}} & =\frac{1}{2}\left\|u_{0}\right\|^{2}-\int_{[T / 3, T / 2] \cap E_{3}} \frac{F\left(t, s u_{0}(t)\right)}{s^{2}} d t-\int_{[0, T] \backslash\left([T / 3, T / 2] \cap E_{3}\right)} \frac{F\left(t, s u_{0}\right)}{s^{2}} d t \\
& \leq \frac{1}{2}-\int_{[T / 3, T / 2] \cap E_{3}} \frac{F\left(t, s u_{0}(t)\right)}{\left|s u_{0}(t)\right|^{2}} d t+m_{1} \int_{0}^{T}\left|u_{0}\right|^{2} d t+\frac{m_{2} T}{s^{2}} \\
& \rightarrow-\infty \quad \text { as } s \rightarrow+\infty,
\end{aligned}
$$

where $E_{3}$ refers to (2.12). Hence, there is $s_{0}>\max \{1, \rho\}$ so large such that $\varphi\left(s_{0} u_{0}\right)<0$. Therefore, by Proposition 2.1, $\varphi$ possesses one nontrivial critical point. This completes the proof.

\section{Proofs of Theorems 1.5 and 1.6}

In this section, we investigate the existence of infinitely many solutions of problem (1.3) under the symmetric assumption upon $F$. by

Under condition (A), the energy functional associated to problem (1.3) given

$$
\psi(u)=\frac{1}{2} \int_{0}^{T}|\dot{u}(t)|^{2} d t-\frac{1}{2} \int_{0}^{T}(A(t) u(t), u(t)) d t-\int_{0}^{T} F(t, u(t)) d t
$$

is continuously differentiable and weakly lower semi-continuous on $H_{T}^{1}$, and

$$
\left\langle\psi^{\prime}(u), v\right\rangle=\int_{0}^{T}(\dot{u}(t), \dot{v}(t)) d t-\int_{0}^{T}(A(t) u(t), v(t)) d t-\int_{0}^{T}(\nabla F(t, u(t)), v(t)) d t
$$

for all $u, v \in H_{T}^{1}$. The critical points of $\psi$ correspond to the weak solutions of problem (1.3) (see [10]).

By the spectral theorem for compact self-adjoint operators on a Hilbert space, the differential operator $u \mapsto-\ddot{u}-A(t) u$ has a sequence of eigenfunctions which forms an orthogonal basis of $H_{T}^{1}$ and the following decomposition holds:

$$
H_{T}^{1}=H^{+} \bigoplus H^{-} \bigoplus H^{0}
$$


where

$$
\begin{aligned}
& H^{+}=\overline{\operatorname{span}\left\{u \in H_{T}^{1}:-\ddot{u}-A(t) u=\lambda u \text { with } \lambda>0\right\}}, \\
& H^{-}=\operatorname{span}\left\{u \in H_{T}^{1}:-\ddot{u}-A(t) u=\lambda u \text { with } \lambda<0\right\}, \\
& H^{0}=\operatorname{ker}\{\ddot{u}+A(t) u\} .
\end{aligned}
$$

One has $\operatorname{dim}\left(H^{-} \oplus H^{0}\right)<+\infty$ and there exists a constant $\delta>0$ such that

$$
\int_{0}^{T}|\dot{u}|^{2} d t-\int_{0}^{T}(A(t) u, u) d t \geq \delta\|u\|^{2}
$$

for $u \in H^{+}$and

$$
\int_{0}^{T}|\dot{u}|^{2} d t-\int_{0}^{T}(A(t) u, u) d t \leq-\delta\|u\|^{2}
$$

for $u \in H^{-}$(see [10, p. 89]).

We shall apply the Fountain Theorem of Bartsch (see [36, Theorem 2.5]) to prove Theorems 1.5 and 1.6. Let $X$ be a reflexive and separable Banach space, then there are $\left(e_{n}\right)_{n \in \mathbb{N}} \subset X$ and $\left(e_{n}^{*}\right)_{n \in \mathbb{N}} \subset X^{*}$ (the dual space of $X$ ) such that

$$
X=\overline{\operatorname{span}\left\{e_{n}: n \in \mathbb{N}\right\}}, \quad X^{*}=\overline{\operatorname{span}\left\{e_{n}^{*}: n \in \mathbb{N}\right\}}
$$

and

$$
\left(e_{n}, e_{m}\right)= \begin{cases}1, & n=m \\ 0, & n \neq m\end{cases}
$$

Let $X_{j}=\operatorname{span}\left\{e_{j}\right\}$, then $X=\overline{\bigoplus_{j \geq 1} X_{j}}$. Now we define

$$
Y_{k}=\bigoplus_{j=1}^{k} X_{j} \quad \text { and } \quad Z_{k}=\overline{\bigoplus_{j \geq k} X_{j}}
$$

Then we have the following Fountain Theorem.

Proposition 3.1. Assume that $\Psi \in C^{1}(X, \mathbb{R})$ satisfies the Cerami condition $(C)$, $\Psi(-u)=\Psi(u)$. For almost every $k \in \mathbb{N}$, there exist $\rho_{k}>r_{k}>0$ such that

(i) $b_{k}:=\inf _{u \in Z_{k},\|u\|=r_{k}} \Psi(u) \rightarrow+\infty$ as $k \rightarrow \infty$;

(ii) $a_{k}:=\max _{u \in Y_{k},\|u\|=\rho_{k}} \Psi(u) \leq 0$.

Then $\Psi$ has a sequence of critical values tending to $+\infty$.

Lemma 3.1 (see [21]). For the Hilbert space $H_{T}^{1}$, define $Y_{k}$ and $Z_{k}$ as in (3.2), then we have

$$
\beta_{k}:=\sup _{u \in Z_{k},\|u\|=1}\|u\|_{\infty} \rightarrow 0 \quad \text { as } k \rightarrow \infty
$$


Proof. The proof follows the same lines as that of [37, Lemma 3.8], since the embedding of $H_{T}^{1}$ into $C\left(0, T ; \mathbb{R}^{N}\right)$ is compact.

Proof of Theorem 1.5. According to Lemma 2.2 and the evenness of $\psi$, we know that $\psi$ satisfies the $(\mathrm{C})$ condition and $\psi(-u)=\psi(u)$. It remains to verify the conditions $(i)$ and (ii) of Proposition 3.1.

Verification of $(i)$. Set $r_{k}=\beta_{k}^{-1}$. By Lemma 3.1, we get

$$
r_{k} \rightarrow+\infty \quad \text { as } k \rightarrow \infty \text {. }
$$

So choosing $k$ sufficiently large such that

$$
Z_{k} \subset H^{+} \text {and } r_{k} \geq\left(4 \delta^{-1} \max _{s \in[0,1]} a(s) \int_{0}^{T} b(t) d t\right)^{1 / 2},
$$

we obtain, using condition $(A)$ and (3.1),

$$
\begin{aligned}
\psi(u) & \geq \frac{\delta}{2}\|u\|^{2}-\int_{0}^{T} F(t, u) d t \\
& \geq \frac{\delta}{2}\|u\|^{2}-\max _{s \in\left[0,\|u\|_{\infty}\right]} a(s) \int_{0}^{T} b(t) d t \\
& \geq \frac{\delta}{2}\|u\|^{2}-\max _{s \in\left[0, \beta_{k}\|u\|\right]} a(s) \int_{0}^{T} b(t) d t \\
& \geq \frac{\delta r_{k}^{2}}{4}
\end{aligned}
$$

for $u \in Z_{k}$ with $\|u\|=r_{k}$. This yields that

$$
\inf _{u \in Z_{k},\|u\|=r_{k}} \psi(u) \geq \frac{\delta r_{k}^{2}}{4} \rightarrow+\infty \quad \text { as } k \rightarrow \infty .
$$

Verification of $(i i)$. Assume that $(i i)$ does not hold for some given $k$. Then there exists a sequence $\left(u_{n}\right) \subset Y_{k}$ such that

$$
\left\|u_{n}\right\| \rightarrow \infty \quad \text { and } \quad \psi\left(u_{n}\right) \geq 0 .
$$

Let $v_{n}=u_{n} /\left\|u_{n}\right\|$. Then $\left\|v_{n}\right\|=1$. Since $\operatorname{dim} Y_{k}<+\infty$, up to a subsequence, we may assume that

$$
v_{n} \rightarrow v_{0} \text { in } Y_{k} \text { and } v_{n}(t) \rightarrow v_{0}(t) \text { a.e. } t \in[0, T]
$$

for some $v_{0} \in Y_{k} \backslash\{0\}$. From $\left(F_{8}\right)$, we obtain, for $t \in E_{3} \cap\left\{t \in[0, T]: v_{0}(t) \neq 0\right\}$,

$$
\frac{F\left(t, u_{n}(t)\right)}{\left|u_{n}(t)\right|^{2}}\left|v_{n}(t)\right|^{2} \rightarrow+\infty \text { as } n \rightarrow \infty,
$$

where $E_{3}$ refers to (2.12). Then, using $\left(F_{7}\right)$ and Fatou's lemma (see [33]),

$$
\begin{aligned}
\int_{0}^{T} \frac{F\left(t, u_{n}\right)}{\left\|u_{n}\right\|^{2}} d t & =\int_{E_{3} \cap\left\{v_{0} \neq 0\right\}} \frac{F\left(t, u_{n}\right)}{\left|u_{n}\right|^{2}}\left|v_{n}\right|^{2} d t+\int_{[0, T] \backslash\left(E_{3} \cap\left\{v_{0} \neq 0\right\}\right)} \frac{F\left(t, u_{n}\right)}{\left\|u_{n}\right\|^{2}} d t \\
& \geq \int_{E_{3} \cap\left\{v_{0} \neq 0\right\}} \frac{F\left(t, u_{n}\right)}{\left|u_{n}\right|^{2}}\left|v_{n}\right|^{2} d t-m_{1} \int_{0}^{T}\left|v_{n}\right|^{2} d t-\frac{m_{2} T}{\left\|u_{n}\right\|^{2}} \\
& \rightarrow+\infty \text { as } n \rightarrow \infty .
\end{aligned}
$$


It follows from the continuity of $A(\cdot)$ that there exists $G>1$ such that

$$
\left|\int_{0}^{T}(A(t) u, u) d t\right| \leq G \int_{0}^{T}|u|^{2} d t, \quad \forall u \in H_{T}^{1}
$$

Combining this with (3.4), we obtain

$$
\begin{aligned}
\psi\left(u_{n}\right) & \leq \frac{1}{2} \int_{0}^{T}\left|\dot{u}_{n}\right|^{2} d t+\frac{G}{2} \int_{0}^{T}\left|u_{n}\right|^{2} d t-\int_{0}^{T} F\left(t, u_{n}\right) d t \\
& \leq\left\|u_{n}\right\|^{2}\left(\frac{G}{2}-\int_{0}^{T} \frac{F\left(t, u_{n}\right)}{\left\|u_{n}\right\|^{2}} d t\right) \\
& \rightarrow-\infty
\end{aligned}
$$

a contradiction with (3.3).

Proof of Theorem 1.6. The proof of Theorem 1.6 is similar to that of Theorem 1.5 with Lemma 2.2 replaced by Lemma 2.3.

\section{References}

[1] A. Ambrosetti, P.H. Rabinowitz, Dual variational methods in critical point theory and applications, J. Functional Analysis 14 (1973) 349-381.

[2] G. Bonanno, R. Livrea, Multiple periodic solutions for Hamiltonian systems with not coercive potential, J. Math. Anal. Appl. 363 (2010) 627-638.

[3] G. Chen, S. Ma, Periodic solutions for Hamiltonian systems without Ambrosetti-Rabinowitz condition and spectrum 0, J. Math. Anal. Appl. 379 (2011) 842-851.

[4] G. Fei, On periodic solutions of superquadratic Hamiltonian systems, Electron. J. Differential Equations, 8 (2002) 12pp.

[5] G. Fei, S.-K. Kim, T. Wang, Periodic solutions of classical Hamiltonian systems without Palais-Smale condition, J. Math. Anal. Appl. 267 (2002) 665678.

[6] X. He, X. Wu, Periodic solutions for a class of nonautonomous second order Hamiltonian systems, J. Math. Anal. Appl. 341 (2008) 1354-1364.

[7] Q. Jiang, C.-L. Tang, Periodic and subharmonic solutions of a class of subquadratic second-order Hamiltonian systems, J. Math. Anal. Appl. 328 (2007) 380-389.

[8] A.C. Lazer, E.M. Landesman, D.R. Meyers, On saddle point problems in the calculus of variations, the Ritz algorithm, and monotone convergence, J. Math. Anal. Appl. 52 (1975) 594-614.

[9] X. Li, J. Su, R. Tian, Multiple periodic solutions of the second order Hamiltonian systems with superlinear terms, J. Math. Anal. Appl. 385 (2012) 1-11. 
[10] J. Mawhin, M. Willem, Critical point theory and Hamiltonian systems, Springer-Verlag, New York, 1989.

[11] S. Ma, Y. Zhang, Existence of infinitely many periodic solutions for ordinary p-Laplacian systems, J. Math. Anal. Appl. 351 (2009) 469-479.

[12] P.H. Rabinowitz, Periodic solutions of Hamiltonian systems, Comm. Pure Appl. Math. 31 (1978) 157-184.

[13] M. Schechter, Nonautonomous second order Hamiltonian systems, Pacific J. Math. 251 (2011) 431-452.

[14] X.H. Tang, J. Jiang, Existence and multiplicity of periodic solutions for a class of second-order Hamiltonian systems, Comput. Math. Appl. 59 (2010) 36463655.

[15] C.-L. Tang, X.-P. Wu, Some critical point theorems and their applications to periodic solution for second order Hamiltonian systems, J. Differential Equations 248 (2010) 660-692.

[16] Z.-L. Tao, C.-L. Tang, Periodic and subharmonic solutions of second-order Hamiltonian systems, J. Math. Anal. Appl. 293 (2004) 435-445.

[17] Z.-L. Tao, S. Yan, S.-L. Wu, Periodic solutions for a class of superquadratic Hamiltonian systems, J. Math. Anal. Appl. 331 (2007) 152-158.

[18] X. Wu, Saddle point characterization and multiplicity of periodic solutions of non-autonomous second-order systems, Nonlinear Anal. 58 (2004) 899907.

[19] Y.-W. Ye, C.-L. Tang, Periodic solutions for some nonautonomous second order Hamiltonian systems, J. Math. Anal. Appl. 344 (2008) 462-471.

[20] Y.-W. Ye, C.-L. Tang, Periodic and subharmonic solutions for a class of superquadratic second order Hamiltonian systems, Nonlinear Anal. 71 (2009) 2298-2307.

[21] Y. Ye, C.-L. Tang, Infinitely many periodic solutions of nonautonomous second order Hamiltonian systems, in press.

[22] Q. Zhang, C. Liu, Infinitely many periodic solutions for second order Hamiltonian systems, J. Differential Equations 251 (2011) 816-833.

[23] Y. Zhang, S. Ma, Some existence results on periodic and subharmonic solutions of ordinary p-Laplacian systems, Discrete Contin. Dyn. Syst. Ser. B 12 (2009) 251-260.

[24] F. Zhao, J. Chen, M. Yang, A periodic solution for a second-order asymptotically linear Hamiltonian system, Nonlinear Anal. 70 (2009) 4021-4026.

[25] F. Zhao, X. Wu, Saddle point reduction method for some non-autonomous second order systems, J. Math. Anal. Appl. 291 (2004) 653-665. 
[26] F. Zhao, X. Wu, Existence and multiplicity of periodic solution for nonautonomous second-order systems with linear nonlinearity, Nonlinear Anal. 60 (2005) 325-335.

[27] W. Zou, S. Li, Infinitely many solutions for Hamiltonian systems, J. Differential Equations 186 (2002) 141-164.

[28] M. Schechter, W. Zou, Weak linking theorems and Schrödinger equations with critical Sobolev exponent, ESAIM Control Optim. Calc. Var. 9 (2003) 601-619.

[29] M. Schechter, Minimax systems and critical point theory, Birkhäuser, Boston, 2009.

[30] M. Struwe, Variational methods, Springer-Verlag, Second Edition, 1996.

[31] M. Izydorek, J. Janczewska, Homoclinic solutions for a class of the second order Hamiltonian systems, J. Differential Equations 219 (2005) 375-389.

[32] D.G. Costa, C.A. Magalhães, Variational elliptic problems which are nonquadratic at infinity, Nonlinear Anal, 23 (1994) 1401-1412.

[33] K. Yosida, Functional Analysis, sixth edition, Springer-Verlag, Berlin, 1980.

[34] P.H. Rabinowitz, Minimax methods in critical point theory with applications to differential equations. CBMS Reg. Conf. Ser. Math., vol. 65, American Mathematical Society, Providence, RI, 1986.

[35] P. Bartolo, V. Benci, D. Fortunato, Abstract critical point theorems and applications to some nonlinear problems with "strong" resonance at infinity, Nonlinear Anal. 7 (1983) 981-1012.

[36] T. Bartsch, Infinitely many solutions of a symmetric Dirichlet problem, Nonlinear Anal. 20 (1993) 1205-1216.

[37] M. Willem, Minimax Theorems, Birkhäuser, Boston, 1996.

School of Mathematics and Statistics, Southwest University,

Chongqing 400715, PR China

and

College of Mathematics Science, Chongqing Normal University,

Chongqing 401331, PR China

E-mail address: yeyiwei2011@126.com

School of Mathematics and Statistics, Southwest University,

Chongqing 400715, PR China

Tel.:+86 23 68253135; fax:+86 23 68253135. E-mail address: tangcl@swu.edu.cn 